\title{
Ecotoxicidade de nanocatalisadores de óxidos de ferro, produzidos a partir da drenagem ácida de mina, quando submetidos à ação de ozônio em meio aquoso
}

\author{
Ecotoxicity of iron oxide nanocatalysts, produced from acid mine \\ drainage, when submitted to the action of ozone in aqueous medium \\ Thalita Grando Rauen ${ }^{1 *} \odot$, Gidiane Scaratti ${ }^{2} \odot$, Reginaldo Geremias ${ }^{2} \odot$, \\ Regina de Fátima Peralta Muniz Moreira² ${ }^{2}$
}

\begin{abstract}
RESUMO
Óxidos de ferro recuperados da drenagem ácida de minas representam uma matéria-prima potencial para a produção de baixo custo de nanogoetita ou nanohematita, com grau de pureza adequado para o seu uso como catalisador em processos de tratamento de efluentes líquidos com ozônio. Assim, a toxicidade das nanopartículas de ferro precisa ser determinada para prever seu impacto no meio ambiente, antes e depois de terem sido utilizadas nesses processos. Nesse contexto, o objetivo deste estudo foi avaliar a toxicidade de nanogoetita e nanohematita produzidas a partir da drenagem ácida de minas bem como comparar os resultados com hematita sintética de alta pureza. A nanogoetita foi obtida da drenagem ácida de minas e, após seu tratamento térmico a $450^{\circ} \mathrm{C}$, produziu nanopartículas de hematita. Os materiais foram caracterizados por difração de raios $X$, microscopia eletrônica de transmissão e determinação da área superficial específica e porosidade com base nas isotermas de adsorção/dessorção de $\mathrm{N}_{2}$. Foram realizados os ensaios de ecotoxicidade usando os protocolos padronizados para bioluminescência com Vibrio fischeri, letalidade da Artemia sp., germinação de sementes de Lactuca sativa L. (alface) e crescimento das raízes de Allium cepa L. (cebola). Os resultados de toxicidade indicaram estabilidade das nanopartículas, que não são alteradas significativamente pela ação do ozônio em meio aquoso. Para todas as amostras, os valores indicaram baixa ou nenhuma toxicidade nas condições dos experimentos, para os bioindicadores utilizados. Esses resultados fornecem indicação de que as nanopartículas de ferro recuperadas da indústria de resíduos podem ser usadas como catalisadores sem efeitos adversos ao meio ambiente.
\end{abstract}

Palavras-chave: nanogoetita; nanohematita; resíduo industrial; toxicidade aguda.

\begin{abstract}
Iron oxides recovered from acid mine drainage represent a potential raw material for the low-cost production of nanogoethite or nanohematite with a degree of purity suitable for its use as a catalyst in processes for treating liquid effluents with ozone. Thus, the toxicity of iron nanoparticles needs to be determined to predict their impact on the environment, before and after they have been used in these processes. In this context, the objective of this study was to evaluate the toxicity of nanogoethite and nanohematite produced from acid mine drainage as well as to compare the results with high-purity synthetic hematite. Nanogoethite was obtained from acid mine drainage and, after its heat treatment at $450^{\circ} \mathrm{C}$, produced nanoparticles of hematite. The materials were characterized by $\mathrm{X}$-ray diffraction, transmission electron microscopy, and determination of the specific surface area and porosity based on $\mathrm{N}_{2}$ adsorption/desorption isotherms. Ecotoxicity tests were carried out using standardized protocols for bioluminescence with Vibrio fischeri, lethality of Artemia sp, germination of Lactuca sativa L (lettuce) seeds, and growth of Allium cepa L (onion) roots. The toxicity results indicated stability of the nanoparticles, which are not significantly altered by the action of ozone in aqueous medium. For all samples, the values indicated low or no toxicity under the conditions of the experiments. These results provide an indication that the iron nanoparticles recovered from the waste industry can be used as catalysts without adverse effects on the environment.
\end{abstract}

Keywords: nanogoethite; nanohematite; industrial waste; acute toxicity.

Universidade Tecnológica Federal do Paraná - Curitiba (PR), Brasil. 2Universidade Federal de Santa Catarina - Florianópolis (SC), Brasil.

*Autor correspondente: tgrauen@utfpredu.br

Conflitos de interesse: os autores declaram não haver conflitos de interesse

Financiamento: Conselho Nacional de Desenvolvimento Científico e Tecnológico (CNPq) - Pós-Doutorado Sênior - processo no 160722/2013-6.

Recebido: 04/05/2O2O - Aceito: 19/11/2O2O - Reg. ABES: 20200162 


\section{INTRODUÇÃO}

Nanopartículas (NPs) inorgânicas são largamente utilizadas em várias áreas da indústria e em inúmeras aplicações médicas (MELO et al., 2016; GILLISPIE et al., 2019; GROSSY et al., 2019; HUANG et al.,2019; INOUÉ et al., 2019; WANG et al., 2020). As nanopartículas de ferro (Fe-NPs), por exemplo, compõem diversos materiais, tanto como componentes principais como combinadas com outros compostos (PAUNOVIC et al., 2020). Das aplicações mais conhecidas, as Fe-NPs têm sido utilizadas no tratamento de águas e solo contaminados. Atuam como adsorventes de metais pesados e catalisadores eficientes de poluentes orgânicos, usando ozônio ou peróxido de hidrogênio em escala de laboratório (OLIVEIRA et al., 2016; SANTOS et al, 2018; GONÇALVES et al., 2019). Poluentes orgânicos, como hidrocarbonetos policíclicos aromáticos, inseticidas e vários compostos orgânicos halogenados, podem ser degradados por meio de processos de oxidação/redução com essas NPs.

O alto custo para produzir NPs à base de ferro puro limitou por muito tempo a aplicação em larga escala para o tratamento de água e efluentes, mas o uso de matérias-primas residuais de atividades industriais são uma alternativa para a produção econômica desses materiais.

Nanogoetita (GO) e nanohematita utilizados neste trabalho são óxidos de ferro já produzidos a partir da drenagem ácida de mina de carvão (DAM) (FLORIANI et al., 2010; FLORES et al, 2012; MADEIRA et al., 2012). O uso dessa matéria-prima residual abriu a possibilidade de produção de Fe-NPs para atender ao mercado da área de tratamento de efluentes industriais. A atividade catalítica desses materiais para aplicação em processos de peroxidação (FLORES et al., 2012) ou ozonização (NOGUEIRA, 2014; MOREIRA et al., 2017) na degradação de poluentes orgânicos dissolvidos em água é comparativamente superior àquela produzida a partir de matérias-primas não residuais.

Várias novas aplicações ambientais de Fe-NP em processos oxidativos avançados, tais como a ozonização, o processo Fenton ou a peroxidação catalítica, comprovaram o uso eficiente desses materiais (SUAVE et al., 2014; DOS SANTOS et al., 2019), indicando que a liberação desses materiais no meio ambiente pode se tornar um grande problema no futuro próximo. Em geral, nos processos de tratamento de águas e efluentes líquidos utiliza-se grande concentração de sólidos (LEI et al, 2016), o que pode resultar em elevada quantidade desses materiais descartada no meio ambiente. Além disso, outra fonte de contaminação ambiental resulta do uso de Fe-NP na biomedicina (BAALOUSHA, 2009; DWIVEDI \& MA, 2014). O depósito desse sólido em aterros sanitários ou no meio ambiente pode causar a sua interação com todos os tipos de compostos ali presentes, orgânicos ou não, dependendo das condições ambientais.

O impacto ambiental causado pelo lançamento não controlado de Fe-NP deve ser investigado, pois a passagem facilitada por meio de barreiras biológicas, devido ao seu menor tamanho, possibilita o seu acúmulo em vários tecidos e células, além de interação com diferentes tipos de compostos (DURÁNVALDEIGT ESIAS et al., 2016; ZHU et al., 2017; EFRAMOVA et al, 2018; LI et al., 2018; SIDIROUPOLOU et al., 2018; HURTADO-GALLEGO et al., 2020; PAUNOVIC et al., 2020; SAYADI et al., 2020).

As características físico-químicas podem influenciar sua toxicidade. As principais, que podem conferir diferenças na toxicidade são o tamanho, a fase cristalina, a forma e a área superficial (PAUNOVIC et al., 2020). Quanto menor o tamanho das Fe-NPs, maior sua toxicidade e interferência no crescimento de algas Chlorella pyrenoidosa (LEI et al., 2016). Entretanto, os estudos de toxicidade têm se limitado à avaliação da toxicidade de Fe-NP sem terem sido previamente submetidas à presença de oxidantes fortes, tais como o ozônio e o peróxido de hidrogênio na toxicidade, tal como são utilizadas no tratamento de águas e efluentes líquidos.

Alguns trabalhos reportados na literatura, utilizando outros níveis tróficos (espécies fitoplanctônicas marinhas Nannochloropsis sp. e Isocirose sp), demonstraram que NPs de $\alpha-\mathrm{Fe}_{2} \mathrm{O}_{3}$ e $\gamma$ - $\mathrm{Fe}_{2} \mathrm{O}_{3}$ apresentaram toxicidade, com supressão significativa do crescimento populacional devido à cobertura superficial das espécies de algas com Fe-NPs, mesmo em baixas concentrações (1 mg.L.-1) (DEMIR et al., 2015). As NPs de $\alpha-\mathrm{Fe}_{2} \mathrm{O}_{3}$ mostraram menor toxicidade que as de $\gamma-\mathrm{Fe}_{2} \mathrm{O}_{3}$, e essa diferença foi atribuída às diferentes distribuições dos poros, da estrutura cristalina e da opacidade em suspensão. Por outro lado, Lei et al. (2016) verificaram que, para a alga de água doce Chlorella pyrenoidosa, as NPs $\gamma-\mathrm{Fe}_{2} \mathrm{O}_{3}$ foram menos tóxicas do que as $\alpha-\mathrm{Fe}_{2} \mathrm{O}_{3}$. Assim, evidencia-se que as condições físico-químicas do meio podem influenciar o potencial tóxico de uma NP.

Quanto aos seres humanos, os óxidos de ferro têm sido amplamente utilizados como agentes de contraste na ressonância magnética ou em veículos derivados de drogas, justamente devido à sua baixa toxicidade (KARLSSON et al, 2015). Porém, já foi observada a supressão da função imunizadora das células T por NPs de óxidos de ferro (LI et al., 2018).

A literatura reporta que várias interações químicas com esses oxidantes podem modificar o estado de oxidação e outras características superficiais (GHASEMPOUR et al., 2015). Consequentemente, são necessários estudos adicionais para avaliar o potencial tóxico desses nanocatalisadores após o seu uso em processos oxidativos avançados.

Assim, o objetivo do presente trabalho foi comparar de toxicidade de NPs de goetita e hematita $(\mathrm{HC})$, submetidas à simulação de um Processo Oxidativo Avançado (POA), a fim de verificar se o ozônio, $\mathrm{O}_{3}$, pode alterar as propriedades das NPs e influenciar sua toxicidade.

\section{METODOLOGIA}

\section{Caracterização de materiais}

GO recuperada de DAM e HC foram cedidas pelo Laboratório de Estudos do Meio Ambiente (LEMA) da Universidade Federal de Santa Catarina (UFSC), Brasil, e da SSNano (Houston, TX, EUA), respectivamente.

NPs de HC (H450) foram produzidas a partir do tratamento térmico da GO a $450^{\circ} \mathrm{C}$, sob atmosfera de ar, resultando NPs denominadas H450.

Os materiais sólidos foram caracterizados por difração de raios $\mathrm{X}$ em um difratômetro de raios X Philips X'Pert., usando fonte de radiação $\mathrm{Cu} \mathrm{K} \alpha$ $(\lambda=1,542 \AA)(40 \mathrm{kV}, 30 \mathrm{~mA})$. As análises foram realizadas a $25^{\circ} \mathrm{C}$ na faixa angular de $0^{\circ}$ a $80^{\circ}(2 \theta)$. As varreduras foram realizadas em etapas de $0,05^{\circ}$, com um tempo de gravação de 1 segundo para cada etapa. A análise morfológica foi executada por meio de microscopia eletrônica de transmissão (TEM) usando um equipamento JEOL JEM-1011 TEM.

A caracterização textural foi realizada mediante adsorção-dessorção de nitrogênio a $77 \mathrm{~K}$ em um adsorptômetro automático Quantachrome Autosorb-1 (EUA), para determinação da área superficial $\mathrm{BET}^{1}$ e do volume de microporos.

${ }^{1} \mathrm{O}$ nome do método é derivado dos nomes dos pesquisadores que o desenvolveram — Stephen Brunauer, Paul Hugh Emmett e Edward Teller. 


\section{Exposição de nanopartículas em meio aquoso com injeção de ozônio}

Os experimentos de ozonização foram realizados no modo semibatelada, em um reator de vidro com capacidade de $2,0 \mathrm{~L}(21 \mathrm{~cm}$ de altura e $8 \mathrm{~cm}$ de diâmetro), a temperatura ambiente $\left(23-26^{\circ} \mathrm{C}\right)$. Uma quantidade fixa de $\mathrm{Fe}-\mathrm{NP}$ $\left(0,5\right.$ g.L $\left.\mathrm{L}^{-1}\right)$ foi continuamente agitada por uma hora, para manter a homogeneidade da suspensão. Essa dosagem de catalisador tem sido reportada na literatura como sendo a que maximiza a relação atividade catalítica:consumo de oxidante (NOGUEIRA, 2014; MOREIRA et al., 2017) Em seguida, uma alíquota de $20 \mathrm{~mL}$ (designada como $\mathrm{t}_{\mathrm{i}}$ ) foi retirada do reator para ser submetida aos testes de toxicidade.

Na sequência, o reator foi alimentado continuamente com ozônio a uma vazão de $0,063 \mathrm{~m}^{3} \cdot \mathrm{h}^{-1}$, gerando uma concentração de aproximadamente $8 \mathrm{mg}$ de $\mathrm{O}_{3}$. $\mathrm{L}^{-1}$ na fase líquida. Após uma hora de ozonização além do tempo da saturação da fase líquida, outra alíquota do meio reacional (designada como $t_{\mathrm{f}}$ ) foi recolhida, para ser submetida aos testes de toxicidade.

É importante ressaltar que nenhuma das alíquotas removidas do reator foi submetida a processos de separação das Fe-NP e que todos os ensaios foram realizados em triplicata.

\section{Análise de toxicidade aguda}

Diferentes níveis tróficos padronizados foram utilizados obedecendo aos seguintes protocolos: bioluminescência com Vibrio fischeri (JOHNSON, 2018), letalidade da Artemia sp. (SVENSSON et al., 2005), germinação de sementes de alface Lactuca sativa L. (ANDRADE et al., 2010) e crescimento das raízes de cebola Allium cepa L. (BORTOLOTTO et al., 2009).

\section{Teste de bioluminescência com V. fischeri}

A toxicidade aguda das amostras $\mathrm{t}_{\mathrm{i}} \mathrm{e} \mathrm{t}_{\mathrm{f}}$ foi realizada, de acordo com o protocolo ISO 11348-3: (2007), usando bactérias bioluminescentes V. fischeri liofilizadas, pelo teste de toxicidade aguda Microtox ${ }^{\oplus}$ no equipamento Analyser, modelo M500. O protocolo de diluição utilizado foi $\mathrm{FD}_{1}-\mathrm{FD}_{16}\left(\mathrm{GL}_{1}-\mathrm{GL}_{16}\right)$. O reagente bacteriano emissor de luz especial liofilizado foi do kit Biolux, adquirido da UmWelt (Brasil) e validado por $\mathrm{ZnSO}_{4} \cdot 7 \mathrm{H}_{2} \mathrm{O}$ com $\mathrm{CE}_{50}=4,73 \mathrm{mg} \cdot \mathrm{L}^{-1}$ (entre 3-10 mg. $\mathrm{L}^{-1}$ ). Esse teste mede a inibição da emissão de luz de bactérias bioluminescentes ( $V$. fischeri) causada pelo efeito tóxico dos produtos químicos testados, durante um período de incubação de 15 e 30 minutos a $15^{\circ} \mathrm{C}$. A concentração efetiva a 30 minutos e $15^{\circ} \mathrm{C}\left(\mathrm{CE}_{50}, \mathrm{mg} \mathrm{L}^{-1}\right)$ tem relação inversa com a toxicidade, portanto, adotaram-se os valores do fator de toxicidade (FT), que corresponde à diluição mínima sem efeito tóxico, cujos valores são diretamente proporcionais à toxicidade e numericamente equivalentes ao fator de diluição (NBR 15411-2-2012). Foram quantificados no software Microtox Omni, versão 4.0, com limite de confiança de $95 \%$. Antes da realização do ensaio, o pH de todas as amostras foi ajustado para 6,5-7,5 e foi determinado usando um medidor de $\mathrm{pH}$ do modelo Micronal B474.

\section{Teste de letalidade em microcrustáceo Artemia sp.}

O teste foi realizado de acordo com a metodologia proposta de Svensson et al. (2005), com modificações. Para isso, os cistos de Artemia sp. (50 mg) foram colocados em $100 \mathrm{~mL}$ de solução salina a $2 \%$, por 24 horas, a cerca de $37^{\circ} \mathrm{C}$, em lugar arejado e ao abrigo da luz, para incubação. As diluições (100, 50, 25 e $12,5 \%$ ) de cada amostra foram feitas em placas de múltiplos poços, por 24 horas, em forno a $25 \pm 2^{\circ} \mathrm{C}$ e ao abrigo de luz. Uma solução salina a $2 \%$ foi usada como controle negativo. Ao final da exposição, uma concentração letal média $\left(\mathrm{CL}_{50}\right)$, definida como a concentração na qual ocorreu $50 \%$ de morte dos organismos bioindicadores, foi utilizada para esse fim, pelo método matemático Trimmed Spearman-Karber (HAMILTON et al., 1977), utilizando-se o programa Probitos ${ }^{\circledast}$.

\section{Teste de inibição da germinação de sementes em Lactuca sativa $L$.}

O teste foi realizado conforme proposto por Andrade et al. (2010). As sementes de Lactuca sativa L., obtidas comercialmente, foram colocadas em contato com papel-filtro embebido em $2 \mathrm{~mL}$ de cada amostra e água mineral (controle negativo), acondicionadas em placas de Petri, em forno a $25 \pm 2{ }^{\circ} \mathrm{C}$ e protegidas da luz, durante 72 horas. Em cada placa eram adicionadas dez sementes e os testes foram realizados em triplicata. Após o período de exposição, determinou-se a porcentagem de germinação das sementes e os resultados foram expressos em média \pm desvio padrão.

\section{Teste de inibição do crescimento radicular de Allium cepa $L$.}

O teste foi realizado de acordo com Bortolotto et al. (2009). Para isso, exemplares de Allium cepa L., obtidos de fontes comerciais, tiveram suas raízes removidas e os bulbos $(n=6)$ colocados no topo de tubos cônicos de polietileno de fundo contendo $50 \mathrm{~mL}$ de cada amostra, em um forno a $25 \pm 2{ }^{\circ} \mathrm{C}$ e protegidos da luz. Para o controle negativo, utilizou-se água mineral obtida comercialmente. As amostras eram reabastecidas diariamente, com manutenção da iluminação. Após sete dias de exposição, a inibição do crescimento radicular foi determinada e os resultados foram expressos em média \pm desvio padrão.

\section{Análise estatística}

A análise estatística dos resultados obtidos nos testes com sementes de Lactuca sativa L. e Allium cepa L. foi realizada por meio da análise de variância (ANOVA), complementada pelos testes de Student-Newman-Keuse e Dunnet, quando necessário. Para tanto, utilizou-se o software INSTAT (GrahPad, San Diego, CA, EUA), assumindo nível de significância de $\mathrm{p}<0,05$.

\section{RESULTADOS E DISCUSSÃO}

\section{Caracterização das Fe-NP}

A Figura 1 mostra a isoterma de adsorção-dessorção de $\mathrm{N}_{2}$ para GO, $\mathrm{H} 450$ e HC. Todas as amostras exibem isotermas do tipo IV, de acordo com a classificação da União Internacional de Química Pura e Aplicada (IUPAC) (GREG \& SING, 1982), indicando que as Fe-NPs têm estrutura mesoporosa (PRÈLOT et al., 2003; ANDERSEN et al., 2012; FLORES et al., 2012).

Os parâmetros da caracterização físico-química e textural das Fe-NPs são apresentados na Tabela 1.

H450 tem área superficial ligeiramente superior à do precursor GO, uma vez que o tratamento térmico de GO produz a desidroxilação da goetita e, consequentemente, aumenta a quantidade de microporos que contribuem para o aumento da área superficial e do volume de poros (LIU et al., 2013). O diâmetro médio dos poros (Tabela 1) confirmou a predominância dos mesoporosos nas três amostras utilizadas neste trabalho (NAMANE et al., 2005). 


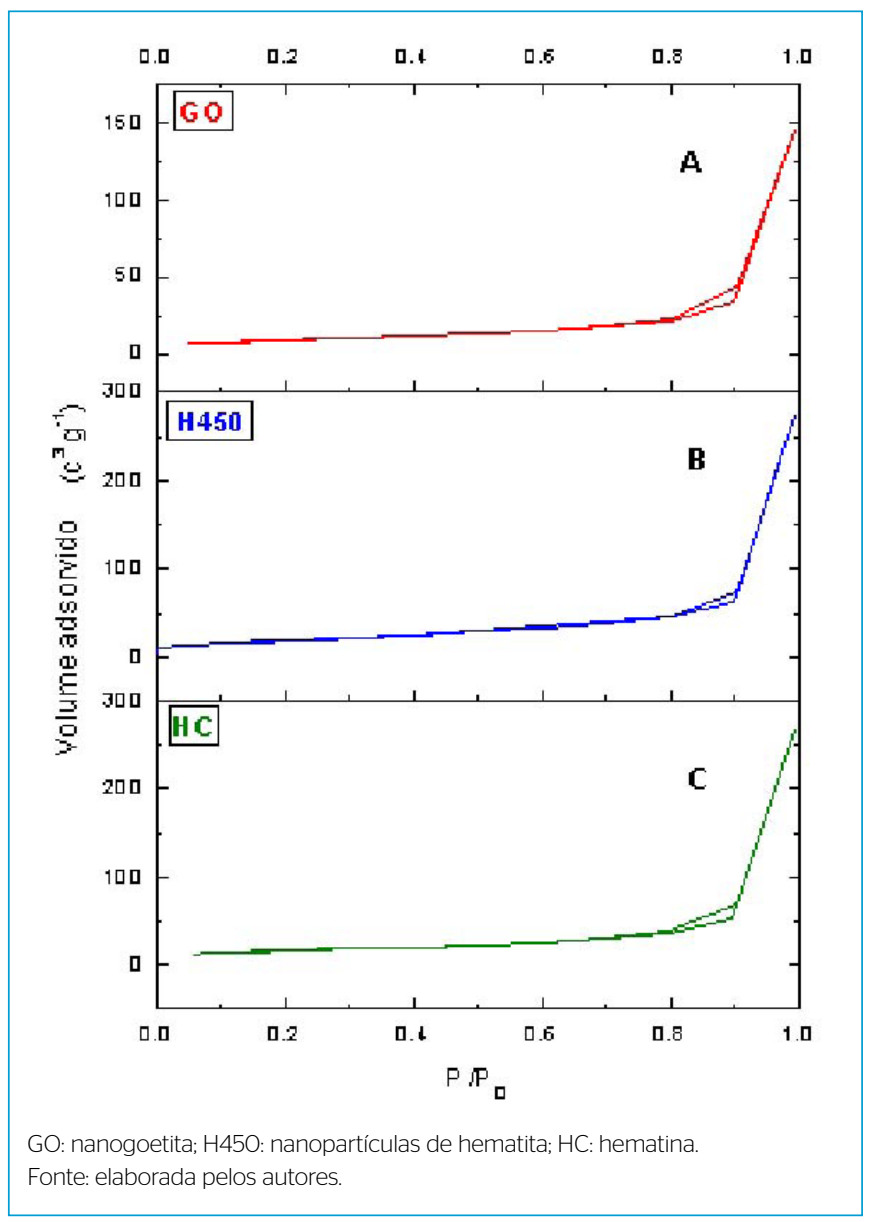

Figura 1 - Isotermas de adsorção-dessorção de $\mathrm{N}_{2}$ para (A) Nanogoethite; (B) nanopartículas de hematita; $\mathrm{e}(\mathrm{C})$ hematina.

Tabela 1 - Parâmetros de caracterização físico-química e textural das nanopartículas de ferro.

\begin{tabular}{l|c|c|c} 
& $\mathrm{GO}$ & $\mathrm{H} 450$ & $\mathrm{HC}$ \\
\hline Area superficial $\mathrm{BET}, \mathrm{m}^{2} \cdot \mathrm{g}^{-1}$ & 58,6 & 66,8 & 31,5 \\
\hline Volume de poros, $\mathrm{cm}^{3} \cdot \mathrm{g}^{-1}$ & 0,41 & 0,43 & 0,22 \\
\hline Diâmetro médio de poros, $\mathrm{nm}$ & 28,2 & 25,5 & 28,6 \\
\hline Dimensões, $\mathrm{nm}$ & $350 \times 30^{\mathrm{a}}$ & $520 \times 363^{\mathrm{b}}$ & $20-40^{\mathrm{c}}$ \\
\hline Morfologia & Acicular & Acicular & Esférica \\
\hline Fase cristalina & Goetita & Hematita & Hematita \\
\hline Tamanho do cristalito, $\mathrm{nm}$ & $18,2^{\mathrm{a}}$ & $13,1^{\mathrm{d}}$ & $10,1^{\mathrm{b}}$ \\
\hline Ponto de carga zero, $\mathrm{pHpcz}$ & $3,1 \pm 0,5^{\mathrm{a}}$ & $5,5 \pm 0,5^{\mathrm{b}}$ & $6,0 \pm 0,5^{\mathrm{b}}$ \\
\hline
\end{tabular}

GO: nanogoetita; H45O: nanopartículas de hematita; HC: nanohematita comercial; a: Scaratti et al., 2017; b: Nogueira, 2014; c: Ssnano, Inc., 2014; ¿: Andersen, et al., 2012. Fonte: elaborada pelos autores

A partir da TEM (Figura 2), determinaram-se a morfologia e o tamanho aproximado das Fe-NPs, que são mostrados na Tabela 1. As NPs de goetita (GO) são aciculares, com diâmetro de $30 \mathrm{~nm}$ e comprimento igual a $350 \mathrm{~nm}$. Embora o comprimento seja superior a $100 \mathrm{~nm}$, ainda podem ser classificadas como NPs por apresentarem dimensão (diâmetro) menor do que 100 nm (PAS71, 2011).
O tratamento térmico de $\mathrm{GO}$, que originou a amostra $\mathrm{H} 450$, não produziu alterações na morfologia ou nas dimensões do seu precursor GO (GONZÁLEZ et al., 2000; ALVAREZ et al., 2006; FLORES et al., 2012). Por outro lado, as NPs de HC (Figura 1C) consistem em partículas esféricas aglomeradas com dimensões entre 20 e $40 \mathrm{~nm}$.

Todas as Fe-NPs utilizadas neste trabalho são cristalinas (Figura 3 e Tabela 1), sendo que as NPs de H450, produzidas a partir de GO, mostraram a presença única da fase $\mathrm{HC}$ como resultado da transformação da fase goetita para $\mathrm{HC}$ a $450^{\circ} \mathrm{C}$ (CHEN et al., 2010). As Figuras 3B e 3C mostram cristalinidade ligeiramente mais alta (picos mais finos) da $\mathrm{HC}$ em relação à amostra $\mathrm{H} 450$.

\section{Ensaios de toxicidade aguda}

A toxicidade das Fe-NPs depende das suas características físico-químicas, dimensões e carga superficial. Entretanto, não existem estudos que reportem a toxicidade de Fe-NPs depois de terem sido utilizadas em processos de ozonização catalítica para o tratamento de efluentes líquidos.

$\mathrm{Na}$ presença de $\mathrm{O}_{3}$ em meio aquoso formam-se diversos tipos de radicais livres e ânions oxigenados ( $\mathrm{HO}_{\bullet}, \mathrm{O}_{2}^{-}, \mathrm{HO}^{-}$, dentre outros) na superfície de $\mathrm{Fe}-\mathrm{NP}$ (ZHANG \& MA, 2009). Embora esses radicais livres sejam extremamente instáveis e sejam rapidamente destruídos na superfície de Fe-NPs, não existem estudos que avaliam as modificações superficiais e a toxicidade das Fe-NPs provocadas pela ação do ozônio. Em geral, goetita tem maior quantidade de grupos superficiais hidroxila do que outras formas cristalinas dos óxidos de ferro, principal fator que torna a atividade catalítica da goetita superior à de outras formas cristalinas de Fe-NP (ZHANG \& MA, 2009). As tabelas apresentam os resultados da análise de toxicidade da suspensão aquosa contendo Fe-NP antes e após uma hora de exposição ao ozônio.

A concentração usual de NPs utilizadas em processos catalíticos par nanorremediação é de 0,5 g.L $\mathrm{L}^{-1}$. A fim de verificar se o descarte do material, após submetido a um POA, comprometeria o ambiente, avaliou-se a toxicidade nos diferentes níveis tróficos apenas nessa concentração.

Os valores obtidos para os diferentes organismos são apresentados nas Tabelas 2, 3, 4 e 5, sendo a bactéria marinha $V$. fischeri e o microcrustáceo marinho Artemia sp. representantes do meio aquático, e Lactuca sativa L. (alface) e Allium cepa $L$. (cebola) vegetais representantes do meio terrestre.

$\mathrm{Na}$ avaliação dos efeitos tóxicos na $V$. fischeri, os valores de $\mathrm{CE}_{50}$, em mg. $\mathrm{L}^{-1}$, para a GO, H450 e HC, antes da ozonização, foram, com intervalo de confiança de 95\%, respectivamente 312,3 (293,1-343,84); 158,2 (128,6-194,6); e 273,6 (161,0-405,4). Após uma hora sob ozonização, os valores de $\mathrm{CE}_{50}$, em mg.L $\mathrm{L}^{-1}$, apresentaram os valores $310,0(238,2-402,4) ; 162,1$ (122,7-214,1); e $283,7(239,4-336,2)$, respectivamente. Esses valores têm relação inversa com a toxicidade, sendo, portanto, mais bem visualizados nos valores de FT, e quanto maior o valor de FT, maior a toxicidade.

Verifica-se que, para todas as amostras analisadas, os valores do FT com a V. fischeri foram menores do que 10 , indicando baixa ou nenhuma toxicidade. A H450 e a GO apresentam valor maior de FT em relação à HC, porém esses dados não mostraram diferenças significativas na toxicidade, indicando estabilidade das NPs mesmo após a exposição ao ozônio em meio aquoso.

Para Artemia sp., nas condições dos testes, não foi observada mortalidade nas diferentes diluições das amostras; sendo assim, o cálculo da mortalidade $\mathrm{CL}_{50}$ não pôde ser realizado e não foi possível concluir a toxicidade para esse microcrustáceo, como mostram os dados da Tabela 3. 


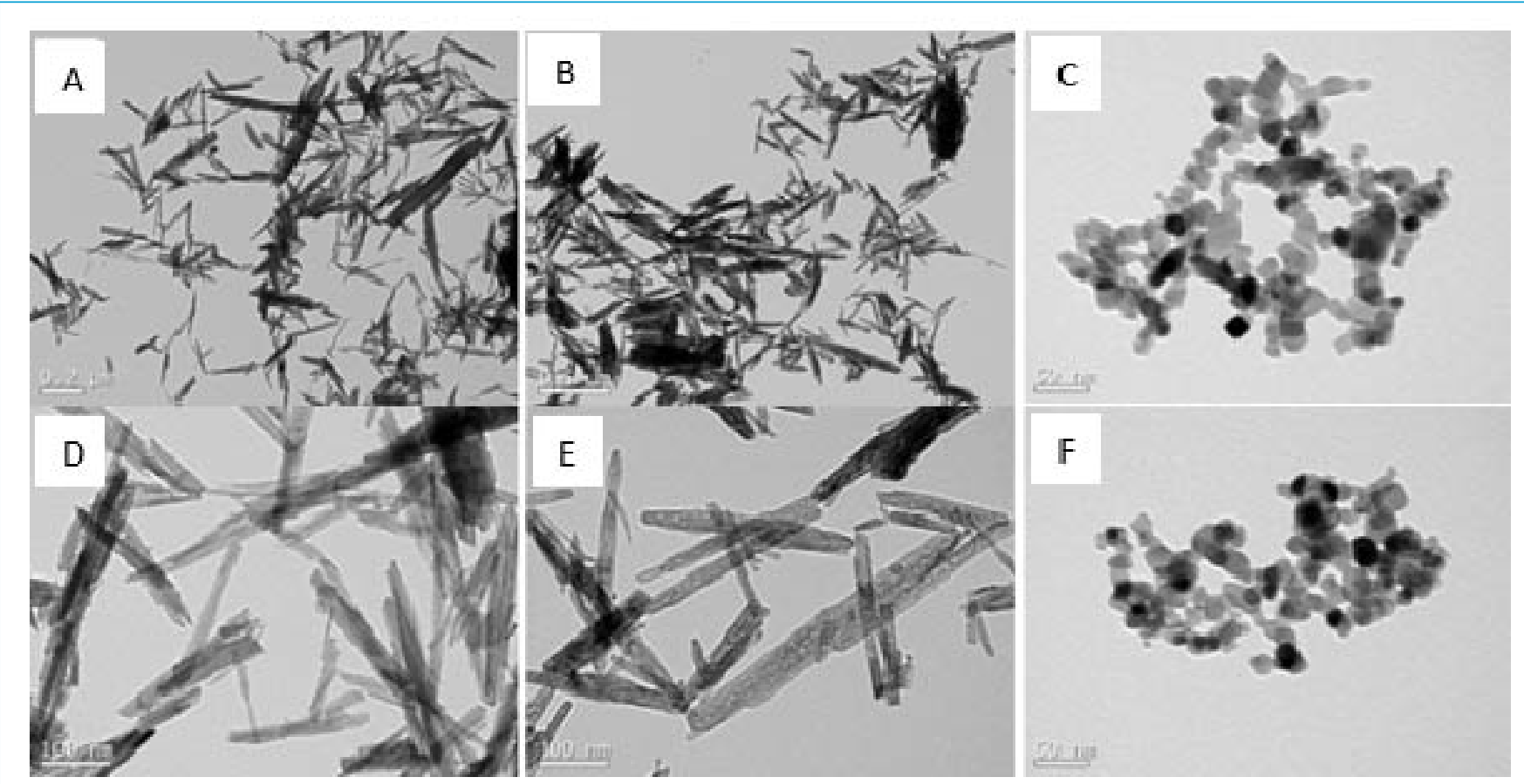

Fonte: elaborada pelos autores.

Figura 2 - Imagens MET de (A) Nanogoethite, (B) nanopartículas de hematita e (C) hematina.

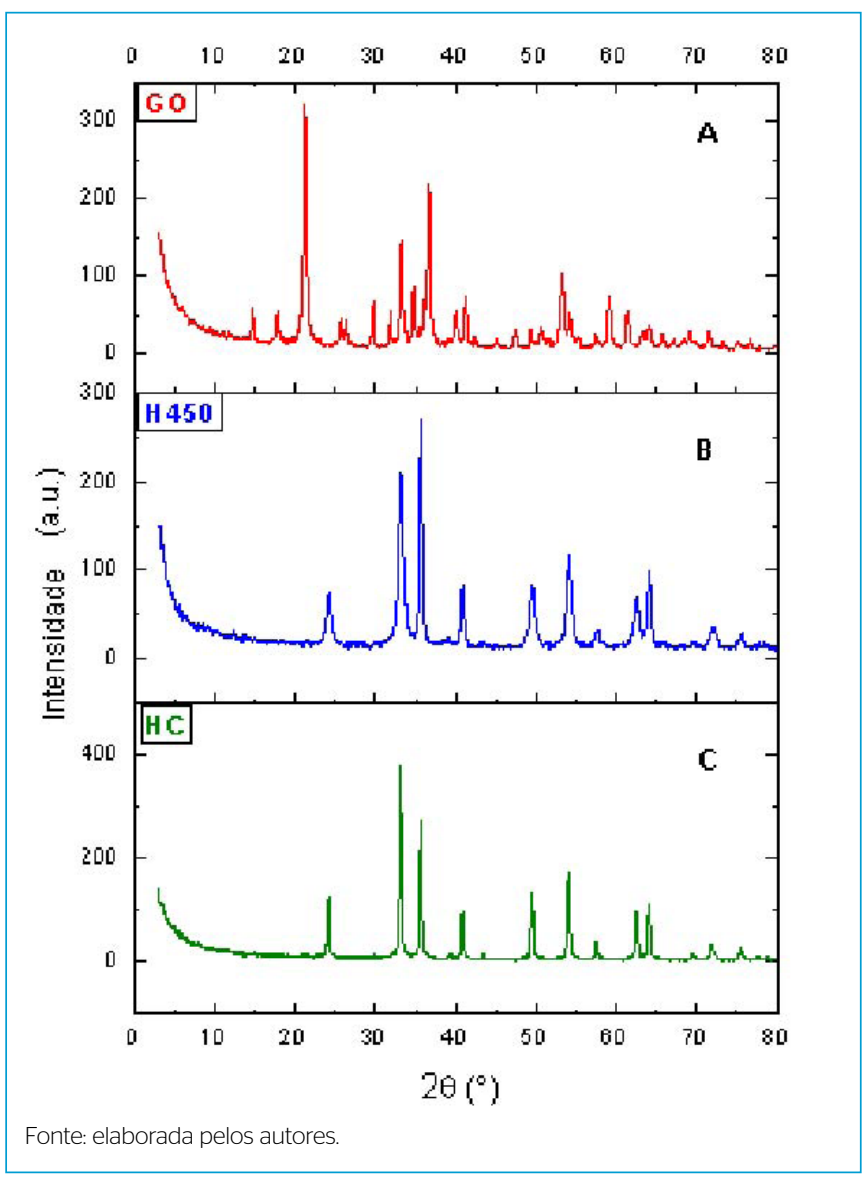

Figura 3 - Difratogramas de difração de raios-X de (A) GO - nanogoetita; (B) H45O - nanohematita recuperada e (C) $\mathrm{HC}$ - nanohematita comercial.
Tabela 2 - Fator de toxicidade de nanopartículas de óxidos de ferro nas bactérias $V$. Fischeri antes de serem submetidas ao ozônio e após uma hora no reator sob ozonização pelo método Microtoxò

\begin{tabular}{l|c|c}
\multirow{2}{*}{} & \multicolumn{2}{|c}{$\mathrm{GL}$} \\
\cline { 2 - 3 } $\mathrm{GO}$ & $\mathrm{ti}$ & tf \\
\hline $\mathrm{H} 450$ & 4 & 4 \\
\hline $\mathrm{HC}$ & 4 & 4 \\
\hline
\end{tabular}

Método Microtox®, protocolo de diluição $\mathrm{GL}_{1}-\mathrm{GL}_{16}$, ISO 11348-3 (2007).

GO: nanogoetita; $\mathrm{H} 45 \mathrm{O}$ : nanopartículas de hematita; $\mathrm{HC}$ : nanohematita comercial; ti: tempo zero, antes de serem submetidas ao ozônio; tf: tempo após uma hora de ozonização.

Fonte: elaborada pelos autores.

Tabela 3 - Mortalidade da Artemia sp. em diferentes diluições da amostra*.

\begin{tabular}{l|c|c} 
& ti & tf \\
$\mathrm{GO}$ & 0 & 0 \\
\hline $\mathrm{H} 450$ & 0 & 0 \\
\hline $\mathrm{HC}$ & 0 & 0
\end{tabular}

*Diferentes diluições da amostra (100, 50, 25 e 12,5\%); GO: nanogoetita; H45O: nanopartículas de hematita; $\mathrm{HC}$ : nanohematita comercial; ti: tempo zero, antes de serem submetidas ao ozônio; tf: tempo após uma hora de ozonização. Fonte: elaborada pelos autores.

Os resultados da avaliação da toxicidade na germinação das sementes de alface e cebola demonstram que as amostras também não causaram inibição significativa da germinação das sementes e raízes, respectivamente, quando comparadas ao controle negativo (água mineral) nas condições dos testes. 
Tabela 4 - Inibição da germinação de sementes de Lactuca sativa L. (\%).

\begin{tabular}{l|c|c} 
(a) & ti & tf \\
\hline $\mathrm{GO}$ & $100 \pm 0$ & $100 \pm 0$ \\
\hline $\mathrm{H} 45 \mathrm{O}$ & $100 \pm 0$ & $90 \pm 0$ \\
\hline $\mathrm{HC}$ & $97 \pm 6$ & $97 \pm 6$ \\
\hline
\end{tabular}

Controle negativo: 100\% \pm 0; GO: nanogoetita; H45O: nanopartículas de hematita: HC: hematita comercial; ti: tempo zero, antes de serem submetidas ao ozônio; tf: tempo após uma hora de ozonização.

Fonte: elaborada pelos autores.

Tabela 5 - Inibição do crescimento de raízes de Allium cepa $L$.

\begin{tabular}{l|c|c} 
(b) & ti & tf \\
$\mathrm{GO}$ & $2,8 \pm 1,6$ & $3,3 \pm 1,8$ \\
\hline $\mathrm{H} 450$ & $3,5 \pm 1,0$ & $3,9 \pm 0,9$ \\
\hline $\mathrm{HC}$ & $3,3 \pm 0,9$ & $3,5 \pm 0,6$ \\
\hline
\end{tabular}

Controle negativo: 5,6 \pm 2,3; GO: nanogoetita; H450: nanopartículas de hematita; HC: nanohematita comercial; ti: tempo zero, antes de serem submetidas ao ozônio; tf: tempo após uma hora de ozonização.

Fonte: elaborada pelos autores.

Os dados de todos os ensaios ecotoxicológicos, nas condições de teste deste trabalho, evidenciam que essas NPs não apresentam efeito tóxico direto nos diferentes níveis tróficos avaliados, tendo sido submetidas ou não ao tratamento por ozonização. Possivelmente, a inibição ao estresse oxidativo nos representantes estudados, na concentração de estudo, foi eficiente para não desenvolver efeitos tóxicos pronunciados.

A avaliação do efeito de substâncias químicas tóxicas sobre organismos representativos de ecossistemas diferentes permite a prevenção do aporte dessas substâncias no ambiente, uma vez que a toxicidade pode se manifestar em diferentes níveis de organização, desde estruturas celulares até indivíduos, populações e comunidades (RONCO et al., 2004).

Estudos do efeito tóxico em representantes diferentes do meio aquático e de vegetais verificaram disfunções celulares em carpas (Cyprinus carpio) (HAO et al., 2009); má formação de embriões e genotoxicidade em células branquiais e sanguíneas de zebrafish (Danio rerio) (ZHU et al., 2012; BATISTA-GALLEP et al., 2018); e inibição de enzimas em embriões medaka (Oryzias latipes) (LI et al., 2009). Em plantas, observou-se redução do desenvolvimento da cultura de rabanetes (Raphanus sativus) (ZUVERZA-MENA et al., 2017); em tomates (Solanumlyco persicum), observou-se estresse hídrico devido à fixação das $\mathrm{Np}-\mathrm{Fe}$ nas raízes (MARTÍNEZ-FENÁNDEZ \& KOMÁREK, 2016). Contudo, esses estudos verificaram que as taxas de toxicidade foram significativas em concentrações superiores às desta pesquisa.

Além disso, não foi observada correlação entre as propriedades físico-químicas e morfológicas das NPs GO, H450 e HC e a toxicidade. Fe-NP com alto grau de pureza tem mostrado baixa citotoxicidade (GARCIA et al., 2011), embora o estresse oxidativo e a inflamação tenham sido observados em ratos (PETTIBONE et al., 2008; SINGH et al., 2008). Os resultados obtidos neste trabalho demonstram que mesmo as Fe-NPs produzidas a partir da DAM apresentam baixa citotoxicidade para os diferentes níveis tróficos utilizados. Sendo assim, os dados podem ser complementares enquanto não há padronização da determinação da avalição dos efeitos tóxicos dessas partículas.

\section{CONCLUSÕES}

A toxicidade de Fe-NPs produzidos a partir de resíduos da DAM foi avaliada e comparada com a toxicidade de Fe-NPs de alta pureza. Os graus de toxicidade em todos os níveis tróficos avaliados não mostraram diferenças significativas na toxicidade dessas amostras. A toxicidade não parece ser afetada pelo processo de ozonização, indicando alguma estabilidade das NPs. Esses resultados fornecem indicação de que as NPs de ferro de resíduos industriais podem ser usadas como catalisadores sem efeitos adversos ao meio ambiente, na dosagem de NPs utilizada neste estudo.

Como os óxidos de ferro são alguns dos óxidos de metais de transição mais importantes para aplicações tecnológicas, incentivar o uso de NPs recuperados de resíduos industriais pode alavancar o aprimoramento das técnicas de produção, tornando a aplicação dessas partículas mais acessível e benéfica ao meio ambiente.

\section{CONTRIBUIÇÃO DOS AUTORES}

Rauen, T. G.: conceituação, curadoria de dados, análise formal, metodologia, escrita — primeira redação, obtenção de financiamento. Scaratti, G.: curadoria de dados, metodologia. Geremias, R.: curadoria de dados, metodologia. Moreira, R.F.P.M.: conceituação, obtenção de financiamento, supervisão, escrita - revisão e edição.

\section{REFERÊNCIAS}

ALVAREZ, M.; RUEDA, E. H.; SILEO, E. E. Structural characterization and chemical reactivity of synthetic Mn-goethites and hematites. Chemical Geology, v. 231, p. 288-299, 2006. https://doi.org/10.1016/J. CHEMGEO.2006.02.003

ANDERSEN, S. L. F.; FLORES, R. G.; MADEIRA, V. S.; JOSÉ, H. J.; MOREIRA, R. F. P. M. Synthesis and characterization of acicular iron oxide particles obtained from acid mine drainage and their catalytic properties in toluene oxidation. Industrial \& Engineering Chemistry Research, v. 51, n. 2, p. 767-774, 2012. http://doi.org/10.1021/ie201269y
ANDRADE, L. F; DAVIDE, L. C; GEDRAITE, L. S. The effect of cyanide compounds, fluorides aluminum, and inorganic oxides present in spent pot liner on germination and root tip cells of Lactuca sativa. Ecotoxicology and Environmental Safety, v. 73, n. 4, p. 626-631, 2010. http://doi.org/10.1016/j. ecoenv.2009.12.012

BAALOUSHA, M. Aggregation and disaggregation of iron oxide nanoparticles: influence of particle concentration, $\mathrm{pH}$ and natural organic matter. Science of the Total Environment, v. 407, n. 6, p. 2093-2101, 2009. https://doi.org/10.1016/j.scitotenv.2008.11.022 
BATISTA-GALLEP, T. B.; PASQUOTO-STIGLIANI, T.; GUILGER, M.; RHEDER, D. T.; GERMANO-COSTA, T.; BILESKY-JOSÉ, N.; FRACETO, L. F.; CARVALHO, C. S.; LIMA, R. Efeitos de nanopartículas comerciais de óxido de ferro $\left(\mathrm{Fe}_{2} \mathrm{O}_{3}\right)$ : citotoxicidade, genotoxicidade e estresse oxidativo. Química Nova, v. 41, n. 9. p. 974-981. 2018. https://doi.org/10.21577/0100-4042.20170271

BORTOLOTTO, T.; BERTOLDO, J. B.; SILVEIRA, F.Z.; DEFAVERI, T. M.; SILVANO, J.; PICH, C. T. Evaluation of the toxic and genotoxic potential of landfill leachates using bioassays. Environmental Toxicology and Pharmacology, v. 28, n. 2, p. 288-293, 2009. https://doi.org/10.1016/j.etap.2009.05.007

GREG, S. J.; SING. K. S. Adsorption surface area and porosity. Londres: Academic Press London, 1982. $141 \mathrm{p}$.

CHEN, Y. H.; LI, F. A. Kinetic study on removal of copper (II) using goethite and hematite nano-photocatalysts. Journal of Colloid and Interface Science, v. 347, n. 2, p. 277-281, 2010. https://doi.org/10.1016/j.jcis.2010.03.050

DEMIR, V.; ATES, M.; ARSLAN, Z:; CAMAS, M.; CELIK, F.; BOGATU, C.; CAN, S. S. Influence of alpha and gamma-iron oxide nanoparticles on marine microalgae species. Bulletin of Environmental Contamination and Toxicology, v. 95, n. 6, p. 725-757, 2015. https://doi.org/10.1007/s00128-015$1633-2$

DOS SANTOS, T. R. A. T., MATEUS, G. O A. O. P., SILVA, M. F.; BERGAMASCO, R. Aplicação da nanotecnologia no tratamento de água: uma revisão. Revista UNINGÁ Review, v. 34, n. 2, p. 51-72, 2019

DURÁN-VALDEIGT ESIAS, E.; ZHANG, W.; NOURY, A.; ALONSO-RAMOS, C.; HOANG, T. H. C.; SERNA, S.; ROUX, X. L.; CASSAN, E.; IZARD, N.; SARTI, F.; TORRINI, U.; BICCARI, F; VINATTIERI, A.; BALESTRIERI, M.; KEITA, A. S.; YANG, H.; BEZUGLY, V.; CUNIBERTI, G.; FILORAMO, A.; GURIOLI, M.; VIVIEN, L. Integration of carbon nanotubes in silicon strip and slot waveguide microring resonators. IEEE Transactions on Nanotechnology, v. 15, n. 4, p. 583-589, 2016. https://doi.org/10.1109/tnano.2016.2556225

DWIVEDI, A. D.; MA, L. Q. Biocatalytic Synthesis Pathways, Transformation, and Toxicity of Nanoparticles in the Environment. Journal Critical Reviews in Environmental Science and Technology, v. 44, n. 15, p. 1679-1739, 2014. https://doi.org/10.1080/10643389.2013.790747

EFREMOVA, M. V.; NAUMENKO, V. A.; SPASOVA, M.; GARANINA, A. S.; ABAKUMOV, M. A.; BLOKHINA, A. D.; MELNIKOV, P. A.; PRELOVSKAYA, A. O.; HEIDELMANN, M.; LI, Z. A.; MA, Z.; SHCHETININ, I. V.; GOLOVIN, Y. I.; KIREEV, I. I.; SAVCHENKO, A. G.; CHEKHONIN, V. P.; KLYACHKO, N. L.; FARLE, M.; MAJOUGA, A. G.; WIEDWALD, U. Magnetite-Gold nanohybrids as ideal all-in-one platforms for theranostics. Scientific Reports, v. 8, n. 1, 11295, 2018. https://doi.org/10.1038/s41598-018-29618-w

FLORES, R. G.; ANDERSEN, S. L. F.; MAIA, L. K. K.; JOSÉ, H. J.; MOREIRA, R. F. P. M. Recovery of iron oxides from acid mine drainage and their application as adsorbent or catalyst. Journal of Environmental Management, v. 111, p. 53-60, 2012. https://doi.org/10.1016/j.jenvman.2012.06.017

FLORIANI, S. L.; VIRMOND, E.; LUIZ, D. B.; ALTHOFF, C. A.; MOREIRA, R. F. P. M.; JOSÉ, H. J. Potential of Industrial Solid Wastes as Energy Sources and Gaseous Emissions Evaluation in a Pilot Scale Burner (ES2008-54355). Journal of Energy Resources Technology, v. 132, n. 1, 2010. https://doi. org/10.1115/1.4001143

GARCIAA, A.; ESPINOSA, R.; DELGADO, L.; CASALS, E.; GONZÁLEZ, E.; PUNTES, V.; BARATA, C.; FONT, X.; SÁNCHEZ, A. Acute toxicity of cerium oxide, titanium oxide and iron oxide nanoparticles using standardized tests, Desalination, v. 269, n. 1-3, p. 136-141, 2011. https://doi.org/10.1016/j. desal.2010.10.052

GHASEMPOUR, S.; SHOKRGOZAR, M. A.; GHASEMPOUR, R.; ALIPOUR, M.; Investigating the cytotoxicity of iron oxide nanoparticles in in vivo and in vitro studies. Experimental and Toxicologic Pathology, v. 67, n. 10, p. 509-515, 2015. https://doi.org/10.1016/j.etp.2015.07.005

GILLISPIE, E. C.; TAYLOR, S. E.; QAFOKU, N. P.; HOCHELLA, M. F. Impact of iron and manganese nano-metal-oxides on contaminant interaction and fortification potential in agricultural systems - a review. Environmental Chemistry, v. 16, n. 6. p. 377-390, 2019. https://doi.org/10.1071/en19063

GONÇALVES, R. G. L.; LOPES, P. A.; RESENDE, J. A.; PINTO, F. G.; TRONTO, J.; GUERREIRO, M. C.; DE OLIVEIRA, L. C. A.; CASTRO NUNES, W.; NETO, J. L. Performance of magnetite/layered double hydroxide composite for dye removal via adsorption, Fenton and photo-Fenton processes. Applied Clay Science, v. 179, p. 105152, 2019. https://doi.org/10.1016/j.clay.2019.105152

GONZÁLEZ, G.; SAGARZAZU, A.; VILLALBA, R. Study of the mechanochemical transformation of goethite to hematite by TEM and XRD. Materia/s Research Bulletin, v. 35, n. 14-15, p. 2295-2308, 2000. https://doi.org/10.1016/ s0025-5408(00)00434-7

GROSSI, J. ; NASCIMENTO, M. A.; OLIVEIRA, A. F.; SILVA, A. A.; LOPES, R. P. The influence of different electrolytes on sulfentrazone dechlorination by iron-nickel bimetallic nanoparticles. Brazilian Journal of Chemical Engineering, v. 36, n. 1, p. 191-200, 2019. https://doi.org/10.1590/01046632.20190361s20170513

HAMILTON, M. A.; RUSSO, R. C.; THURSTON, R. V. Trimmed SpearmanKarbermethod for estimating median lethal concentrations in toxicity bioassays. Environmental Science \& Technology, v. 11, n. 7. p. 714-719, 1977. https://doi.org/10.1021/es60130aO04

HAO, L.; WANG, Z:; XING, B. Effect of sub-acute exposure to $\mathrm{TiO}_{2}$ nanoparticles on oxidative stress and histopathological changes in Juvenile Carp (Cyprinus carpio). Journal of Environmental Science, v. 21, n. 10, p. 14591466, 2009. https://doi.org/10.1016/s1001-0742(08)62440-7

HUANG, W.; JACKSON, M. J.; DEKKERS, M. J.; SOLHEID, P.; ZHANG, B.; GUO, Z:; DING, L. Nanogoethite as a Potential Indicator of Remagnetization in Red Beds. Geophysical Research Letters, v. 46, n. 22, p. 12841-12850, 2019. https://doi.org/10.1029/2019gl084715

HURTADO-GALLEGO, J.; PULIDO-REYES, G.; GONZALEZ-PLEITER, M.; SALAS, G.; LEGANÉS, F.; ROSAL, R.; FERNÁNDEZ-PIÑAS, F. Toxicity of superparamagnetic iron oxide nanoparticles to the microalga Chlamydomonas reinhardtii. Chemosphere, v. 238, p. 124562, 2020. https:// doi.org/10.1016/j.chemosphere.2019.124562

INOUE, S.; YSUHARA, A.; Al, H; HOCHELLA, M. F. J.; MURAYAMA, M. Mn (II) oxidation catalyzed by nanohematite surfaces and manganite/ hausmannite core-shell nanowire formation by self-catalytic reaction Geochimica et Cosmochimica Acta, v. 258, p. 79-96, 2019. https://doi. org/10.1016/j.gca.2019.05.011

ISO. ISO 11348-3, Water quality - determination of the inhibitory effect of water samples on the light emission of Vibrio fischeri (Luminescent bacteria test), Part 3: Method using freeze-dried bacteria. International Organization for Standadization, Geneva, Switzerland, 2007. 
JOHNSON, B. T. Microtox ${ }^{\circledR}$ Toxicity Test System - New Developments and Applications. In: WELLS, P. G.; LEE, K; BLAISE, C. Microscale testing in aquatic toxicology: advances, techniques, and practice. Taylor \& Francis Group, 2018. p. 200-212.

KARLSSON, H. L.; TOPRAK, M. S.; FADEEL, B. Toxicity of metal and metal oxides nanoparticles. In: Nordberg, G. F.; Fowler, B. A.; Nordberg, M. (Eds.). Handbook on the Toxicology of Metals. Academic Press. 4. ed. 2015.

LEI, C.; ZHANG, L.; YANG, K.; ZHU, L.; LIN, D. Toxicity of iron-based nanoparticles to green algae: Effects of particle size, crystal phase, oxidation state and environmental aging, Environmental Pollution, v. 218, p. 505-512, 2016. https://doi.org/10.1016/j.envpol.2016.07.030

LI,X.:WANG, X.; SHA, L.; WANG, D.; SHI, W:ZHAO, Q.; WANG, S. Thermosensitive Lipid Bilayer-Coated Mesoporous Carbon Nanoparticles for Synergistic hermochemotherapy of Tumor. Applied Materials and Interfaces, v. 10, n. 23, p. 19386-19397, 2018. https://doi.org/10.1021/acsami.8b03008

LI, H.; ZHOU, Q.; WU, Y.; FU, J.; WANG, T.; JIANG, G. Effects of waterborne nano-iron on medaka (Oryzias latipes): antioxidant enzymatic activity, lipid peroxidation and histopathology. Ecotoxicology and Environmental Safety, v. 72, n. 3, p. 684-692, 2009. https://doi.org/10.1016/j.ecoenv.2008.09.027

LIU, H.; CHEN, T.; ZOU, X.; QING, C.; FROST, R. L. Thermal treatment of natural goethite: Thermal transformation andphysical properties. Thermochimica Acta, v. 568, p. 115-121, 2013. https://doi.org/10.1016/j.tca.2013.06.027

MADEIRA, V. S.; ANDERSEN, S. L. F.; FLORES, R. G. JOSÉ, H. J.; MOREIRA, R. F. P. M. Synthesis and Characterization of Acicular Iron Oxide Particles Obtained from Acid Mine Drainage and Their Catalytic Properties in Toluene Oxidation. Industrial \& Engineering Chemistry Research, v. 51, n. 2 , p. 767-774, 2012. https://doi.org/10.1021/ie201269y

MARTÍNEZ-FENÁNDEZ, D.; KOMÁREK, M. Structural and ultrastructural changes in nanoparticle exposed plants. Environmental and Experimental Botany, v. 131, 2016.

MELLO, V. S.; SILVA, A. E. D.; GUEDES, A. E. D. S; ALVES, S. M.; CARVAJAL, T. R. Leverage about synthesis and dispersion with $\mathrm{CuO}$ nanoparticle in oil lubricating. Journal of Engineering and Technology for Industrial Applications, v. 2, n. 5, p. 53-60, 2016. https://doi.org/10.5935/24470228.20160008

MOREIRA, R. F. P. M.; BALDISSARELLI, V. Z.; CENTURIAO, A. P. S. L.; SCARATTI, G; AMORIM, S. M. Enhanced ozonation degradation of petroleum refinery wastewater in the presence of oxide nanocatalysts. Environmental Technology, v. 21, p. 1-32, 2017.

NAMANE, A.; MEKARZIA, A.; BENRACHEDI, K.; BELHANECHE-BENSEMRA, N.; HELLAL, A. Determination of the adsorption capacity of activated carbon made from coffee grounds by chemical activation with $\mathrm{ZnCl}_{2}$ and $\mathrm{H}_{3} \mathrm{PO}_{4}$. Journal of Hazardous Materials, v. 119, n. 1-3, p. 189-194, 2005. https:// doi.org/10.1016/j.jhazmat.2004.12.006

NOGUEIRA, M. R. C. Sintese, caracterização e atividade catalitica de nanopartículas de óxidos de ferro para a ozonização de sulfametoxazol. Tese (Doutorado) - Universidade Federal de Santa Catarina, Florianópolis, 2014.

OLIVEIRA, J. S.; DRUMM, F. C.; MAZUTTI, M. A.; FOLETTO, E. L.; JAHN, S. L. Preparação do sistema $\mathrm{Fe}_{2} \mathrm{O}_{3} / \mathrm{ZSM}-5$ para uso como catalisador na reação foto-Fenton. Cerâmica, v. 62, n. 363, p. 281-287, 2016. https://doi. org/10.1590/0366-69132016623632022

PAS 71:2011. Nanoparticles. Vocabulary. Disponível em: https://shop. bsigroup.com/ProductDetail/?pid=000000000030214797. Acesso em: O8 out. 2021.

PAUNOVIC, J.; VUCEVIC, D.; RADOSAVLJEVIC, T.; MANDIĆ-RAJČEVIĆ, S.; PANTIC, I. Iron-based nanoparticles and their potential toxicity: focus on oxidative stress and apoptosis. Chemico-Biological Interactions, v. 316, p. 108935, 2020. https://doi.org/10.1016/j.cbi.2019.108935

PETTIBONE, J. M.; ADAMCAKOVA-DOOD, A.; THORNE, P. S.; O'SHAUGHNESSY, P. T.; WEYDERT, J. A.; GRASSIAN, V. H. Inflammatory response of mice following inhalation exposure to iron and copper nanoparticles. Nanotoxicology, v. 2, n. 4, p. 189-204, 2008. https://doi. org/10.1080/17435390802398291

PRĖLOT, B.; VILLIÉRAS, F.; PELLETIER, M.; GERARD. G.; GABORIAUD. F.; EHRHARDT, J. J.; PERRONE, J.; FEDOROFF, M.; JEANJEAN, J.; LEFEVRE, G.; MAZEROLLES, L.; PASTOL, J. L.; ROUCHAUD, J. C.; LINDECKER, C. Morphology and surface heterogeneities in synthetic goethites. Journal of Colloid and Interface Science, v. 261, n. 2, p. 244-254, 2003. https://doi. org/10.1016/s0021-9797(03)00058-4

RONCO, A.; BAEEZ, M. C. D.; GRANADOS, Y. P. Em Ensayos Toxicológicos y Métodos de Evaluación de Calidad de Aguas. In: MORALES, G. C. (Ed.). Estandarización, Intercalibración, Resultados y Aplicaciones. Ottawa: Centro Internacional de Investigaciones para el Desarrollo, 2004.

SANTOS, M. C. E.; PEREIRA, N. M. S.; LIMA, S. B.; SILVA, M. L. A. Catalisadores baseados em óxidos de ferro suportados em carvão ativado destinados à reação de fenton para degradação de poluentes orgânicos: uma revisão bibliográfica. SEPA-Seminário estudantil de produção acadêmica, v. 12, 243, 2018.

SAYADI, M. H.; MANSOURI, B.; SHAHRI, E.; TYLER, C. R.; SHEKARI, H.; KHARKAN, J. Exposure effects of iron oxide nanoparticles and iron salts in blackfish (Capoeta fusca): Acute toxicity, bioaccumulation, depuration, and tissue histopathology, Chemosphere, v. 247, p. 125900, 2020. https://doi. org/10.1016/j.chemosphere.2020.125900

SIDIROPOULOU, E.; FEIDANTSIS, K; KALOGIANNIS, S.; GALLIOS, G. P.; KASTRINAKI, G.; PAPAIOANNOU, E.; VACLAVÍKOVA, M.; KALOYIANNI, M. Insights into the toxicity of iron oxides nanoparticles in land snails. Comparative Biochemistry and Physiology Part C: Toxicology \& Pharmacology, v. 206-207, p. 1-10, 2018. https://doi.org/10.1016/j. cbpc.2018.02.001

SINGH, M.; SINGH, S.; PRASAD, S.; . Nanotechnology in medicine and antibacterial effect of silver nanoparticles. Digest Journal of Nanomaterials and Biostructures, v. 3, p. 115-122, 2008.

SKYSPRING NANOMATERIALS. Iron Oxide Nanoparticles/Nanopowder (Fe203, alpha, 99\%, 20 40 nm. Disponível em: https://www.ssnano.com/ inc/sdetail/iron-oxide-nanoparticles---nanopowder---fe2o3--alpha--99---2040nm-/308. Acesso em: 08 out. 2021.

SUAVE, J.; JOSÉ, H. J.; MOREIRA, R. F.P. M. Degradation of polyvinylpyrrolidone by photocatalytic ozonation and evaluation of the influence of some operational parameters. Ozone: Science \& Engineering, v. 36, n. 6, p. 560569, 2014. https://doi.org/10.1080/01919512.2014.894452 
SVENSSON, B. M.; MATHIASSON, L.; MÅRTENSSON, L.; BERGSTRÖM, S. Artemia salina as test organism for assessment of acute toxicity of leachate water from landfills. Environmental Monitoring and Assessment, v. 102, n. 1-3, p. 309-321, 2005. https://doi.org/10.1007/s10661-005-6029-z

WANG, L.; SHI, C.: WANG, L.; PAN, L:ZHANG, X:ZOU, J. J. Rational design, synthesis, adsorption principles and applications of metal oxide adsorbents: a review, Nanoscale, v. 12, n. 8, p. 4790-4815, 2020. https://doi.org/10.1039/c9nr09274a

ZHANG, T.; MA, J. Catalytic ozonation of trace nitrobenzene in water with synthetic goethite. Journal of Molecular Catalysis A: Chemical, v. 279, n. 1, p. 82-89, 2008a. https://doi.org/10.1016/..molcata.2007.09.030

ZHU, Y.; WANG, Y.; SHA, L.; ZHAO, J.; XIAOYI, L. Design of antibacterial polyethylene terephthalate master batch functionalized by modified nano-
Mg(OH) 2. Journal of Applied Polymer Science, v. 135, n. 46, p. 46755, 2017. https://doi.org/10.1002/app.46755

ZHU, X.; TIAN, S.; CAI, Z. Toxicity Assessment of Iron Oxide Nanoparticles in Zebrafish (Danio rerio) Early Life Stages. PLoS One, v. 7, n. 9, p. e46286, 2012. https://doi.org/10.1371/journal.pone.0046286

ZUVERZA-MENA, N.; MARTÍNEZ-FERNÁNDEZ, D.; DU, W.; HERNANDEZVIEZCAS, J. A.; BONILLA-BIRD, N.; LOOPEZ-MORENO, M. L.; KOMÁREK, M.; PERALTA-VIDEA, J. R.; GARDEA-TORRESDEY, J. L. Exposure of engineered nanomaterials to plants: insights into the physiological and biochemical responses - a review. Plant Physiology and Biochemistry, v. 110, p. 236-264, 2017. https://doi.org/10.1016/j.plaphy.2016.05.037 